\title{
Transient small-scale brightenings in the quiet solar corona: A model for campfires observed with Solar Orbiter ${ }^{\star}$
}

\author{
Yajie Chen ${ }^{1,2}$, Damien Przybylski ${ }^{2}$, Hardi Peter ${ }^{2}$, Hui Tian ${ }^{1,3}$, F. Auchère ${ }^{4}$, and D. Berghmans ${ }^{5}$ \\ 1 School of Earth and Space Sciences, Peking University, 100871 Beijing, PR China \\ e-mail: chenyajie@pku.edu.cn, huitian@pku.edu.cn \\ 2 Max-Planck Institute for Solar System Research, 37077 Göttingen, Germany \\ 3 Key Laboratory of Solar Activity, National Astronomical Observatories, Chinese Academy of Sciences, Beijing 100012, PR China \\ ${ }^{4}$ Université Paris-Saclay, CNRS, Institut d'Astrophysique Spatiale, 91405 Orsay, France \\ 5 Solar-Terrestrial Centre of Excellence - SIDC, Royal Observatory of Belgium, 1180 Brussels, Belgium
}

Received 23 February 2021 / Accepted 21 April 2021

\begin{abstract}
Context. Recent observations by the Extreme Ultraviolet Imager (EUI) on board Solar Orbiter have characterized prevalent smallscale transient brightenings in the corona above the quiet Sun termed campfires.

Aims. In this study we search for comparable brightenings in a numerical model and then investigate their relation to the magnetic field and the processes that drive these events.

Methods. We used the MURaM code to solve the 3D radiation magnetohydrodynamic equations in a box that stretches from the upper convection zone to the corona. The model self-consistently produces a supergranular network of the magnetic field and a hot corona above this quiet Sun. For the comparison with the model, we synthesized the coronal emission as seen by EUI in its $174 \AA$ channel, isolated the seven strongest transient brightenings, and investigated the changes of the magnetic field in and around these in detail.

Results. The transients we isolated have a lifetime of about 2 min and are elongated loop-like features with lengths around $1 \mathrm{Mm}$ to $4 \mathrm{Mm}$. They tend to occur at heights of about $2 \mathrm{Mm}$ to $5 \mathrm{Mm}$ above the photosphere, a bit offset from magnetic concentrations that mark the bright chromospheric network, and they reach temperatures of above $1 \mathrm{MK}$. As a result, they very much resemble the larger campfires found in observations. In our model most events are energized by component reconnection between bundles of field lines that interact at coronal heights. In one case, we find that untwisting a highly twisted flux rope initiates the heating.

Conclusions. Based on our study, we propose that the majority of campfire events found by EUI are driven by component reconnection and our model suggests that this process significantly contributes to the heating of the corona above the quiet Sun.
\end{abstract}

Key words. Sun: magnetic fields - Sun: corona - magnetohydrodynamics (MHD)

\section{Introduction}

On average, in the solar atmosphere, the temperature increases with height above the chromosphere and reaches $\sim 1 \mathrm{MK}$ in the corona. Clearly, the heating of the upper atmosphere is related to the magnetic field, but how the energy is generated, transported, and dissipated is still under debate (e.g., Aschwanden 2019). Observations in the extreme ultraviolet (EUV) have been used abundantly to study the statistics of heating events as well as individual events to infer properties of the underlying heating process(es).

In terms of imaging, the Extreme-ultraviolet Imaging Telescope (EIT, Delaboudinière et al. 1995), the Transition Region and Coronal Explorer (TRACE, Handy et al. 1999), the Extreme UltraViolet Imager (EUVI; Howard et al. 2008), and the Atmospheric Imaging Assembly (AIA, Lemen et al. 2012) have been used extensively. The latest of these, AIA, achieved a spatial resolution of about $1.4^{\prime \prime}$ or $1000 \mathrm{~km}$ on the Sun (at disk center). In addition, recent rocket flights of the High-resolution Coronal imager (Hi-C; Kobayashi et al. 2014; Rachmeler et al. 2019)

\footnotetext{
$\star$ Movies associated to Figs. 2, 4, 5, and A1 are available at https: //www . aanda.org
}

have achieved a spatial resolution of about $0.4^{\prime \prime}$ to $0.5^{\prime \prime}$, corresponding to about 300 to $350 \mathrm{~km}$ on the Sun. These instruments have been used to investigate the distribution of events in terms of energy deposition, or rather radiative output, and they generally found that this follows a power law with most events happening at small spatial scales (e.g., Berghmans et al. 1998; Harra et al. 2000; Aschwanden \& Parnell 2002; Tiwari et al. 2019). The larger brightenings, for example coronal bright points, that have been spatially resolved often show a temporal evolution, as is expected for reconnection events (e.g., Madjarska 2019). The role of reconnection for transient brightenings is underlined by spectroscopic investigations. These show observational signatures of reconnection in the form of bi-directional flows or extreme line broadening in smallscale brightenings, may it be explosive events (e.g., Dere et al. 1989; Innes et al. 1997; Chen et al. 2019a) or UV bursts (e.g., Peter et al. 2014; Tian et al. 2018; Young et al. 2018).

Such reconnection brightenings observed in (E)UV have been studied in numerous 2D and 3D numerical models based on magnetohydrodynamics (MHD). The 2D models have mostly been tailored to reproduce the observational signatures of reconnection, in particular for explosive events, either 
with an idealized setup of a current sheet (e.g., Innes et al 2015; Ni et al. 2016) or for cases where the magnetic structure is driven from the surface (e.g., Peter et al. 2019; Ni et al. 2021). More comprehensive 3D models could even give a selfconsistent description of UV bursts (Hansteen et al. 2017, 2019). In essentially all of these numerical models about explosive events and UV bursts, inducing both $2 \mathrm{D}$ and 3D models, the reconnection occurs between almost antiparallel magnetic field structures.

Recently, the Extreme Ultraviolet Imager (EUI; Rochus et al. 2020) on board Solar Orbiter (Müller et al. 2020) performed observations of the quiescent corona in the $174 \AA$ passband near the disk center. These data were taken during the first perihelion pass of Solar Orbiter at a distance of $\sim 0.6 \mathrm{AU}$ from the Sun while still being in the commissioning phase. The High-Resolution Imager (HRI) of EUI provided EUV images at a very high spatial resolution of about $400 \mathrm{~km}$ on the Sun. The whole observation sequence with a cadence of $5 \mathrm{~s}$ lasted for less than five minutes, but this was sufficient to study more than a thousand compact loop-like brightenings, now termed campfires (Berghmans et al. 2021). The observational results concerning these campfires can be summarized as follows (based on Berghmans et al. 2021):

1. The distribution of lifetimes is power-law-like with most of the events observed by EUI/HRI lasting for less than 2 min.

2. The bulk of the detected events have linear sizes of below $4 \mathrm{Mm}$ and the sizes follow a power-law-like distribution.

3. Most events are elongated loop-like features with an aspect ratio of length to width ranging from 1 to 5 .

4. They occur at the edges of bright patches of the chromospheric network, that is, close to but not directly above the brightest parts of the network.

5. Triangulation with AIA data revealed a height of these events in the range of $1 \mathrm{Mm}-5 \mathrm{Mm}$ above the photosphere.

6. They reach temperatures between $1 \mathrm{MK}$ and $1.6 \mathrm{MK}$

7. The total radiative losses in the $174 \AA$ channel also follow a power-law-like distribution.

Aspects of these findings have been reported before. For example, Chitta et al. (2021) have recently reported on statistics of small-scale brightenings observed with AIA, which are probably similar in nature to the campfires, but they appear on larger scales. This is clear because the spatial resolution of AIA on the Sun is about a factor of 2.5 worse than EUI at $0.6 \mathrm{AU}$. A spatial resolution comparable to, or slightly better than, EUI has been achieved by Hi-C. With $0.4^{\prime \prime}$ to $0.5^{\prime \prime}$, Hi-C could resolve structures down to $300 \mathrm{~km}$ to $350 \mathrm{~km}$; of course, if it were at future perihelia closer than $0.3 \mathrm{AU}$ to the Sun, EUI would surpass Hi-C. Similar to the campfires, Hi-C could identify tiny loops with lengths as small as $1 \mathrm{Mm}$ and with lifetimes, aspect ratios, and a location with respect to the network similar to campfires (Peter et al. 2013; Barczynski et al. 2017). However, only a handful of these miniature loops have been reported. Observations from IRIS (De Pontieu et al. 2014) and Hi-C have also revealed numerous subarcsec brightenings (e.g., Tian et al. 2014; Alpert et al. 2016). However, these elongated brightenings are mostly seen in transition region lines and no clear coronal response was detected. Combining the results 1 to 7 as listed above, EUI described a feature which, in its completeness, escaped detection through previous instruments.

Our study aims to see if transient coronal brightenings similar to the campfires are also found in a 3D model, and if so, to understand their nature. For this, we constructed a self-consistent and time-dependent quiet-Sun model extending from the upper convection zone to the corona using a realistic $3 \mathrm{D}$ radiation
MHD simulation. We synthesized EUI $174 \AA$ images from the model, that is, as EUI would see our model. Finding campfirelike events, we investigated their thermal properties and associated magnetic field topologies. Our study suggests that these transient brightenings are mostly associated with plasma heating due to component reconnection.

\section{MHD model and coronal emission}

\subsection{Radiation MHD simulation using MURaM}

We performed a 3D radiation MHD simulation using the coronal extension of the MURaM code (Vögler et al. 2005; Rempel 2017). The computational domain covers a region of $50 \times 50 \mathrm{Mm}^{2}$ in the horizontal direction and extends from $\sim 20 \mathrm{Mm}$ below to $\sim 17.5 \mathrm{Mm}$ above the photosphere in the vertical direction. The grid size of the simulation is $\sim 48.8 \mathrm{~km}$ and $25 \mathrm{~km}$ in the horizontal and vertical directions, respectively.

We used periodic boundary conditions in the horizontal direction. The upper boundary condition is open to outflows, closed to inflows, and the magnetic field is specified by a potential boundary condition. The lower boundary condition is open to inflows and outflows, with symmetric magnetic field components (Open-boundary Symmetric-field (OSb) in Rempel 2014). This boundary condition allows inflows to carry magnetic fields near the equipartition field strength into the domain in order to model recirculation deeper in the convection zone. Although a small-scale dynamo can operate locally in the photosphere (Schüssler \& Vögler 2008), a boundary condition that models a deeper convection zone is required to reproduce the observationally inferred quiet-Sun field strengths (Rempel 2014).

The simulation parameters are similar to those described in Rempel (2017) with a few modifications. For the higher resolution simulations presented in this work, the diffusion $h$ parameters were decreased slightly to 1.8 in the photosphere and chromosphere (density $\rho>10^{-10} \mathrm{~g} \mathrm{~cm}^{-3}$ ). In the corona $\left(\rho<10^{-10} \mathrm{~g} \mathrm{~cm}^{-3}\right)$, a value of $h=0.8$ was used for the diffusion of the density, energy, velocity, and $h=3.2$ on the magnetic field. We used gray local-thermal-equilibrium (LTE) radiative transfer in the photosphere and low chromosphere. We used a pre-tabulated equation of state generated using FreeEoS (Irwin 2012) and opacities from MPS-ATLAS (Witzke et al. 2021). From the mid-chromosphere to the corona, we used the tabulated $\mathrm{H}, \mathrm{Ca}$, and $\mathrm{Mg}$ line losses as well as the optically thin losses described by Carlsson \& Leenaarts (2012). In order to prevent double cooling in the chromosphere, we turned off the $3 \mathrm{D}$ radiation scheme at an optical depth of $\tau_{\text {cutoff }}=10^{-5}$ with a function of the form $\tau_{5000}^{2} /\left(\tau_{5000}^{2}+\tau_{\text {cutoff }}^{2}\right)$.

The simulation was initialized with the energy and density profiles from a G2V stellar model, which is constant in the horizontal directions. The simulation initially extends $\sim 1 \mathrm{Mm}$ above the solar surface. A small random velocity field was used to start convection. This setup was run for $53 \mathrm{~h}$ of solar time until convection developed throughout the box. A zero net-flux magnetic field seed field was then added by setting a random vertical field for each horizontal pixel, with a root mean square (rms) value of $10^{-3} \mathrm{G}$, and it is constant in the vertical direction. This setup was run for another $58 \mathrm{~h}$ until the magnetic field strength was saturated. The simulation was then extended $5 \mathrm{Mm}$ further above the surface and run for an hour until a stable transition region formed. Finally, the domain was extended another $11.5 \mathrm{Mm}$ and run for 30 min until the corona was self-consistently heated. We collected snapshots for $16 \mathrm{~min}$ at a cadence of $20 \mathrm{~s}$. 


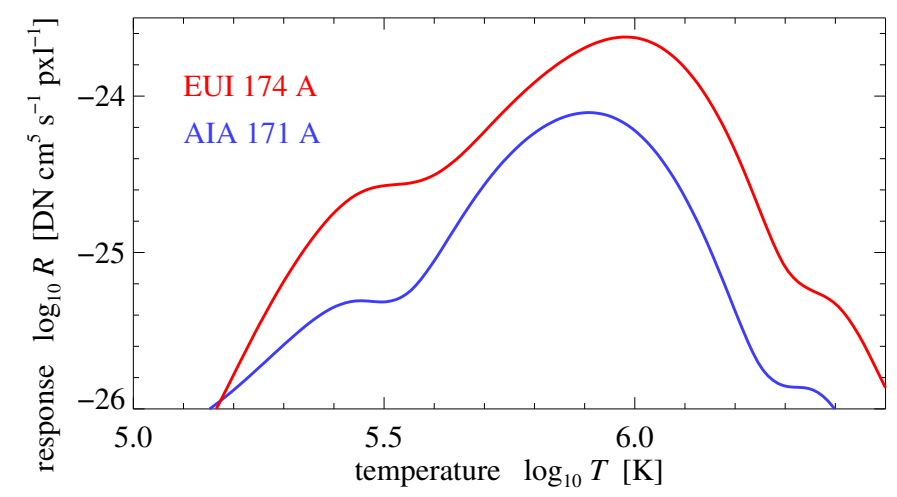

Fig. 1. Temperature response, or contribution, function $R(T, n)$. The red line shows the response for the EUI channel at $174 \AA$, and the blue line corresponds to the one for AIA at $171 \AA$. Both responses are shown here for a density of $n=10^{9} \mathrm{~cm}^{-3}$, which is typical for coronal loops. The radiative losses (per volume) in the respective band are given by $n^{2} R$. See Sect. 2.2.

\subsection{Coronal emission synthesized from the model}

In order to compare our model results with actual coronal observations, we synthesized coronal emission in the EUI $174 \AA$ and AIA $171 \AA$ channels by using response (or contribution) functions $R(T, n)$ that mainly depend on temperature $T$ and to a lesser degree on electron density $n$. In the optically thin corona, where the lines (and continua) are excited by electron collisions, the radiative loss (per unit volume) is then given by $L=n^{2} R(T, n)$.

To determine $R(T, n)$, we first calculated the spectra in the respective wavelength regions using Chianti version 10 (Dere et al. 1997; Del Zanna et al. 2021) with the standard Chianti ionization equilibrium and the coronal abundances of Feldman (1992). We did this for a grid of temperatures and densities; additionally, for each combination of temperatures and densities, we multiplied the resulting spectrum with the effective area (as a function of wavelength) of the respective channel. Integrating over the wavelength with the proper normalization then provides the response function $R(T, n)$. We show the response functions for the EUI $174 \AA$ and AIA $171 \AA$ channels in Fig. 1. In the case of the AIA response, we also considered the degradation of its sensitivity, which is why the AIA response is weaker than the EUI response. The peak in EUI is at slightly higher temperatures because the EUI $174 \AA$ band is dominated by a mix of Fe IX and Fe X, while the AIA $171 \AA$ band is dominated by Fe IX. Also, the contribution from the transition region around a few $10^{5} \mathrm{~K}$ is slightly more pronounced in EUI. Otherwise the two response curves are very similar. The EUI/HRI contribution function used in this Letter is based on a preliminary analysis of the calibration data. The absolute value and wings will likely change somewhat with the final preflight calibration. The central wavelength and overall shape of the peak are, however, not likely to be affected.

We calculated the radiative loss $L$ per unit volume at each grid cell by calculating $R(T, n)$ at that cell and multiplying it with $n^{2}$. To calculate the image as it would be seen by either EUI or AIA, we then simply integrated along the line of sight. In this study we focus on a vertical line of sight, that is, the images we show correspond to actual observations at the disk center. This is because we want to compare our model to the observations presented by Berghmans et al. (2021) that were taken at the disk center.

For better comparison to actual data, we also degrade the coronal images we synthesized from the model to roughly match the spatial resolution of the EUI High Resolution Imager (HRI) at $174 \AA$ and the AIA channel at $171 \AA$. In order to do this, we simply convolved the maps from the model with a symmetric $2 \mathrm{D}$ Gaussian. For the kernels for EUI and AIA, we chose a full width at half maximum of $450 \mathrm{~km}$ and $900 \mathrm{~km}$, respectively, which roughly corresponds to the resolution of the two instruments. At the time of the campfire observations by Berghmans et al. (2021), Solar Orbiter was about 0.6 AU from the Sun. Then two EUI/HRT pixels correspond to about $400 \mathrm{~km}$, so our resolution estimate for EUI here is conservative.

\section{Results}

In order to study transient coronal brightenings and their relation to the magnetic field, we first inspected the coronal images of the 16-minute time series of our numerical model for such events. In Fig. 2 we display a single snapshot that shows the vertical magnetic field at the photosphere, that is, a magnetogram (panel a) and the corresponding coronal image in the $174 \AA$ channel (panel b). Here, one brightening is highlighted by the red box that sits next to a small magnetic field concentration.

From the time series of the synthesized coronal images, we singled out seven individual events for a detailed case study (see Sect. 3.2). Of course, the criterion we chose there is somewhat arbitrary, but we wanted to select a few individual events that we can still study carefully on a case-by-case basis. A future study should look at this, or a similar, model in a statistical way, for example, using the same procedures as in the campfire study by Berghmans et al. (2021). Consequently, we were not be able to recover the power-law-like distributions as found in Berghmans et al. (2021). Instead, we unravel the nature of a selection of the events by investigating changes in the magnetic field during each of the seven events.

In the following, we first compare the properties of the brightenings in our 3D model to the observed campfires (Sect. 3.2). We then investigate the magnetic nature of these brightenings in Sect. 3.3.

\subsection{Magnetic fields in the photosphere}

The photospheric magnetograms in our model show small-scale magnetic concentrations with a field strength on the order of one thousand Gauss and weak salt-and-pepper magnetic elements with a field strength of several hundred Gauss (see Fig. 2a). This is consistent with observations of the quietSun magnetic fields (e.g., Bellot Rubio \& Orozco Suárez 2019). The magnetic fields in our model are maintained through a small-scale dynamo process (Rempel 2014). The magnetic field concentrations form a long-living network pattern. The generation of network and internetwork fields are associated with convection on the supergranule and granule scales. With a horizontal extent of $50 \times 50 \mathrm{Mm}^{2}$, our computational domain is large enough so that several supergranular cells fit into the box.

\subsection{Coronal transient brightenings in the model}

The spatially averaged temperature in the coronal part of our quiet-Sun model is $\sim 1.2 \mathrm{MK}$ and remains stable in time. However, the temperature shows a large spatial variation due to the localization of transient heating events. The local temperature also reveals a large temporal variation because of the intermittency of the energy releases. Consequently, we find significant 

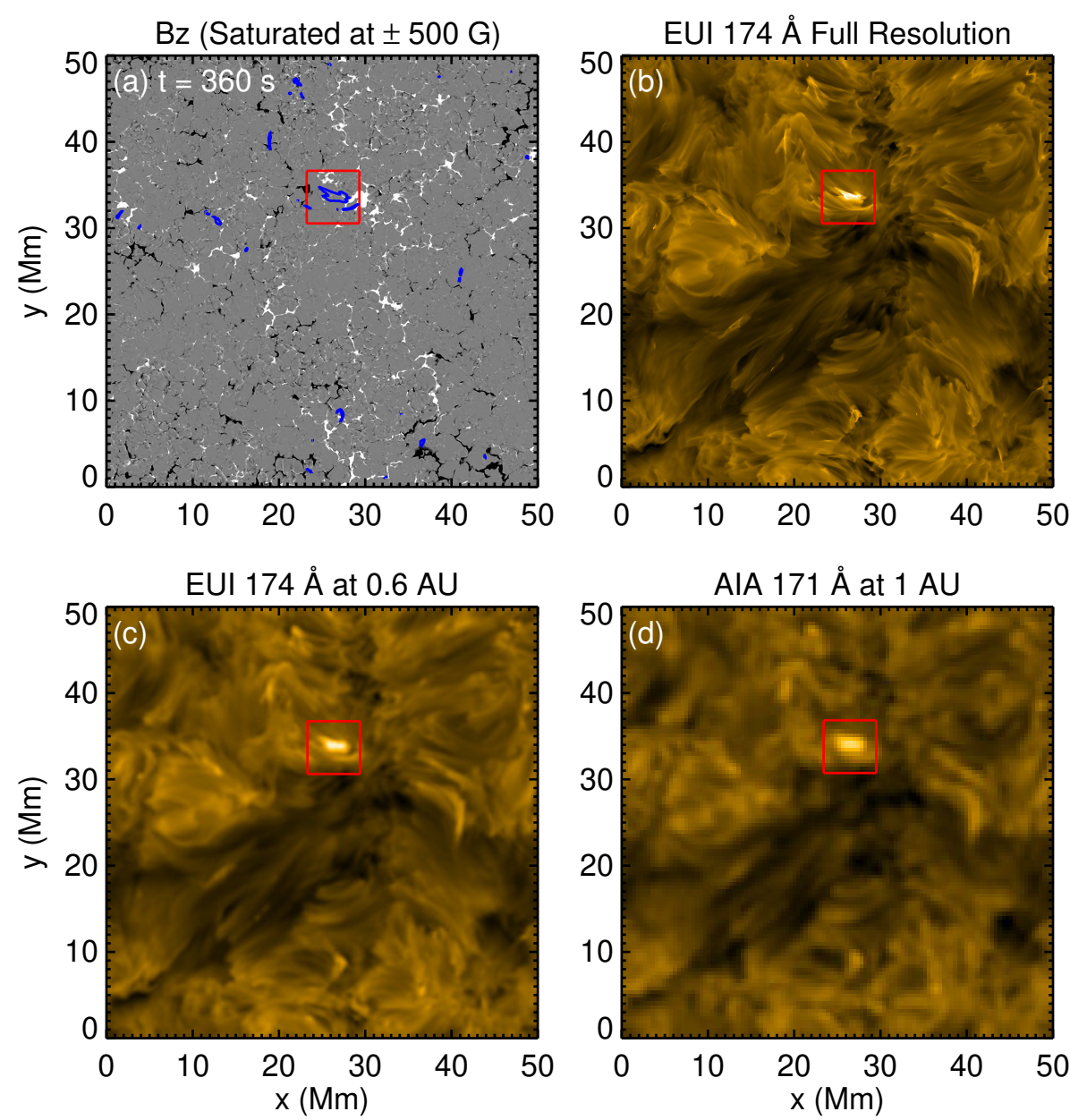

Fig. 2. Photospheric magnetic field and coronal emission in a 3D MHD model. Panel a: vertical component of the photospheric magnetic field saturated at $\pm 500 \mathrm{G}$. This would correspond to a magnetogram at the disk center. Panel b: coronal emission in the $174 \AA$ band integrated along the vertical direction at the full resolution of the numerical model $(50 \mathrm{~km}$ grid spacing in the horizontal directions). Contours of regions with an EUV intensity of $5 \sigma$ above the average intensity are overplotted in blue on the magnetogram in panel a in order to relate bright patches to the magnetic structure. Panel c: same emission in the EUI band as in panel $b$, but now degraded to a spatial resolution similar to EUI at $0.6 \mathrm{AU}$ (ca. $200 \mathrm{~km}$ per pixel plate scale). For comparison, panel $d$ : we show how AIA would see the model at $171 \AA$, that is, we reduced the resolution to match AIA (plate scale of ca. $450 \mathrm{~km}$ per pixel). The EUV images are shown on a logarithmic scale over a dynamic range of 1000 . These panels show the full horizontal extent of the computational domain at one single snapshot. The red box highlights one coronal brightening that is discussed in more detail in Fig. 3. An animation is available online. See Sect. 3.2. fluctuations in the coronal emission as synthesized in the $174 \AA$ band in both space and time.

In order to identify transient brightenings as campfires, we applied the following procedure to the synthetic EUI images reduced to the approximate EUI/HRI resolution. The intensity in a given spatial pixel (in the $x-y$ intensity map) has to be $5 \sigma$ above the mean value over the whole time series. Furthermore, this has to be the case in at least nine contiguous pixels and for at least two snapshots (that are $20 \mathrm{~s}$ apart). If these conditions are met, we call this a campfire, which is the case for seven events in our model data.

We first concentrate on one of these seven events that is highlighted in Fig. 2. In Fig. 3a we show a zoom into this brightening. At the original model resolution, it has a flame-like morphology with a length of about $4 \mathrm{Mm}$ and a width of roughly $1 \mathrm{Mm}$, and it lasts for about $2 \mathrm{~min}$. This is largely consistent with the size, aspect ratio, and lifetime of observed campfires, though this example would be on the large and long-living side (items 1, 2, and 3 in the introduction).

Comparing the location of the sample brightening highlighted in Fig. 2 to the location of the underlying magnetic field shows that it is offset from the magnetic concentration. The zoom in Fig. 3a underlines that the elongated brightening connects the underlying opposite magnetic polarities (shown as contours), but it does not reach them. Therefore, we can also expect this campfire to be offset to the bright chromospheric network because the bright network patches are found directly above the strong magnetic field concentrations (e.g., Barczynski et al. 2018). In Fig. 2a, we mark the coronal $174 \AA$ emission in the magnetogram by contour lines to show how the more numerous smaller brightenings are related to the magnetic field. Just as for the bigger event, these avoid the centers of the network elements, that is, the magnetic concentrations. This is because the field lines are rooted in the magnetic concentrations, but the coronal brightenings happen away from the photospheric footpoints and hence are offset when looking from straight above. This fits well with the location of the observed campfires (item 4 in the introduction).

The brightening of the sample campfire from Fig. 2 occurs at a height of ca. $2 \mathrm{Mm}$ above the photosphere. This can be seen from the vertical cut in Fig. 3b. Other events we looked at in more detail show the bright emission at slightly higher altitudes, for example, the example in Fig. 5 occurs at about $4 \mathrm{Mm}$. Thus the campfires we find in our model occur in the same height range as found in the observations (item 5 in the introduction).

To examine the thermal properties of the sample campfire, we studied vertical cuts through this transient. In Fig. 3 we show the synthesized EUI $174 \AA$ emission, temperature, and density through the middle of the brightening. The temperature and density within the brightening are about $1 \mathrm{MK}$ and $3 \times 10^{10} \mathrm{~cm}^{-3}$. Some plasma embedded in the bright structure was heated to significantly higher temperatures of $3 \mathrm{MK}$. Because of the sharp drop in the response function above $1 \mathrm{MK}$, this does not show up in $174 \AA$ emission. Still, it might show up in EUV channels (e.g., AIA at $211 \AA$ ) or in X-rays probing hotter plasma. It will be interesting to investigate the role of these small-scale brightenings for the quiet-Sun X-ray emission in a future study. For the sample campfire in Fig. 3, we can conclude that it shows plasma 

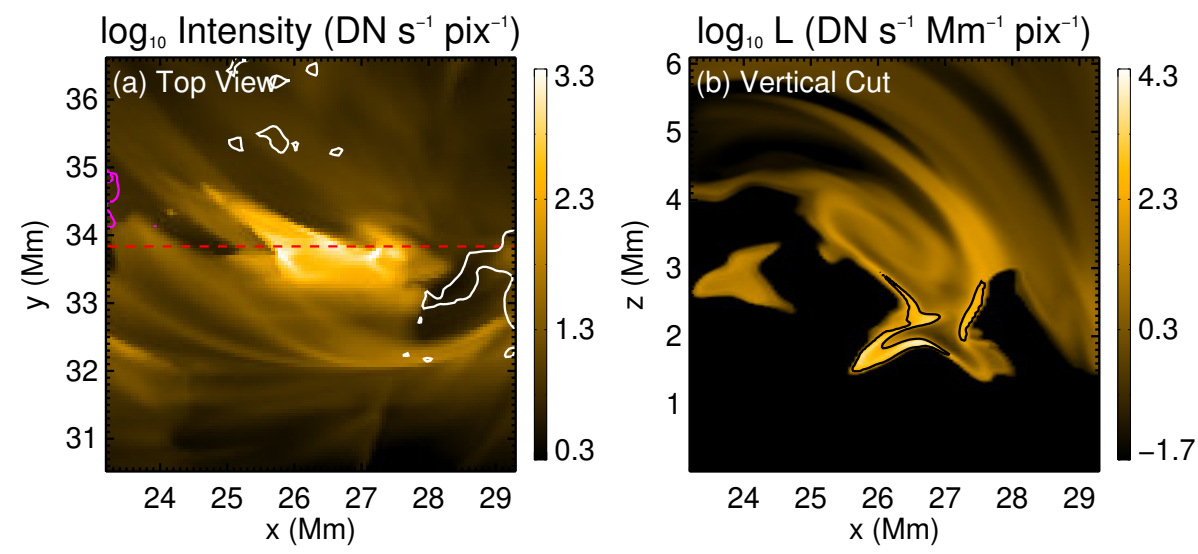

Fig. 3. Thermal structure in a modeled campfire. Panel a: synthesized emission in the EUI $174 \AA$ channel as seen from above. The field of view corresponds to the red box in Fig. 2. The purple and white contours correspond to vertical magnetic fields at $\pm 1000 \mathrm{G}$ in the photosphere. Panels $b-d$ : vertical cuts through the campfire at the $y$ position indicated by the dashed line in panel a. The cut of the radiative losses per volume in the $174 \AA$ band is plotted in panel $b$. The black contours outline the regions of enhanced radiation. These contours are also overplotted on the temperature (panel c) and number density (panel $d$ ) in order to read off the temperature and density in the campfire. The event shown here is the same as the one shown in Fig. 4c. The temporal evolution of the emission from this campfire is displayed in panel 4c of Fig. B.1. See Sect. 3.2.

at around $1 \mathrm{MK}$ and is thus also consistent with the observed campfires (item 6 in the introduction).

In investigating the temporal evolution of the plasma at the location of the transient brightening, we find that the enhancement in EUV radiation is caused by an increase in temperatures. The plasma that is present at the location prior to this campfire gets heated and raises its temperature by more than a factor of ten from below the transition region to coronal temperatures. This results in an expansion of the gas that goes along with a decrease in density.

Finally, we turn to the radiative flux from the campfire. For this, we rebinned the intensity map to the plate scale of EUI (ca. $200 \mathrm{~km}$ at $0.6 \mathrm{AU}$ ) and found peak count rates around $1000 \mathrm{DN}$ per pixel and second. When integrating this over the spatial extent and the lifetime of the brightening, the total number of counts was about $6 \times 10^{5} \mathrm{DN}$. To compare this to the values in Berghmans et al. (2021), we had to convert the DN into detected photons (ca. 6.8 DN per photon) and account for the exposure time $(3 \mathrm{~s})$ being shorter than the image cadence $(5 \mathrm{~s})$. Then, for the sample campfire highlighted in Fig. 2, we found a total of about 50000 photons. This is in the range of the detected photons in observed campfires (Fig. 2 of Berghmans et al. 2021), as is expected for its comparatively large size and long lifetime in the upper range of the observed distribution. Thus this sample model campfire is consistent with observations in terms of radiative output (item 7 in the introduction).

The fact that the sample transients we discuss in this model are on the larger side of the observed campfires is partly due to our selection criteria. We wanted to select a limited number of examples for a case-by-case study and concentrate on the larger events. In addition, the spatial resolution in our numerical model is only a factor of four better than in the observations in terms of grid spacing or pixel size. The smallest, 1-pixel features identified by EUI as campfires might have magnetic sources in the photosphere that might not be resolved in our numerical model. Hence we might miss these smallest events in our model.

The seven campfires we have studied here have common observable signatures. Just as the one example we discussed in detail above, they match the properties of the observed campfires summarized in items 1 to 7 in the introduction. Their lifetimes can be seen from Fig. B.1. Their morphology, in particular length and aspect ratio, is illustrated in Figs. 4, 5, and A.1, and their lifetimes are illustrated in Fig. B.1. From these figures it is also clear that they appear close to magnetic concentrations, but with some offset to the network patches. We illustrate the heights for only two cases (in Figs. 3 and 5), but the others also occur at heights around $2 \mathrm{Mm}$ to $5 \mathrm{Mm}$ above the photosphere. Here, we show data on temperature and radiative losses only for one case, but the others are of the same order of magnitude. Based on this, we can conclude that the seven brightest or strongest coronal transients we find in our numerical model fit the properties of the observed campfires well.

\subsection{Magnetic field in and around the coronal brightenings}

Having established that the transient coronal brightenings in our model match the properties of the observed campfires, the main question is what causes these brightenings in our model. For this we investigate the magnetic field structure in and around these brighenings and, in particular, follow its temporal evolution.

For the analysis of the magnetic field for each of the seven events, we traced field lines crossing the brightening and followed this in time. For each case, we randomly selected 15 to 20 seed points in the region of enhanced EUI $174 \AA$ emission when the campfire was close to its peak intensity (see Appendix B) and traced the magnetic field lines from these points in both 


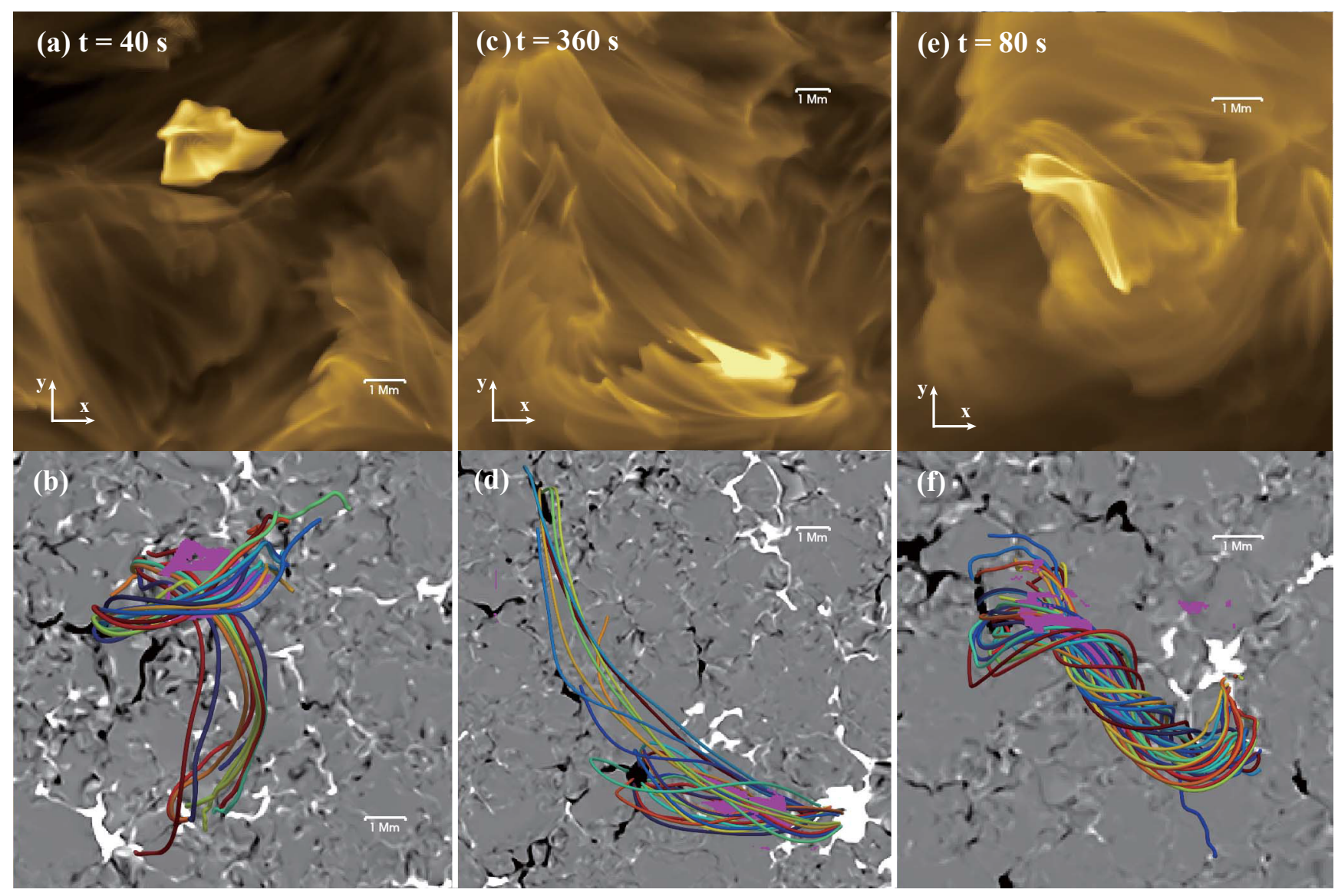

Fig. 4. Campfires and their relation to the magnetic field. Panel $a$ : synthetic EUI 174 Å image of a small field-of-view around a transient brightening close to its maximum intensity (the time given in the panel label; see also light curves in Fig. B.1). The $x$ and $y$ directions are marked and a bar indicates a scale of $1 \mathrm{Mm}$. The intensity scale is logarithmic. Panel $b$ : vertical magnetic field in the photosphere in the same field of view and at the same time as in panel a. The magnetogram is saturated at $\pm 350 \mathrm{G}$. In purple we show a mask of the bright EUV emission in panel a. The differently colored lines show field lines that pass through the volume of the transient brightening (cf. Sect. 3.3). Panels $c-d$ and e-f: same thing as panels $a-b$, just for different campfires. These three examples represent the groups (i) to (iii) defined in Sect. 3.3. More examples are shown in the same format in Figs. 5 and A.1. Animations for these three cases are available online. See Sect. 3.3.

directions. We chose the number of field lines in each case so that it was possible to follow them in the figures; we modified the locations and numbers of the seed points and found that this provides similar results. To capture the evolution of the magnetic field, we used the same seed points to trace field lines in the other snapshots to study the temporal evolution of the magnetic field topologies around the campfires.

We found that the magnetic field structures of the seven identified campfires can be categorized into three groups. For each group we display an example in Fig. 4 showing one snapshot in the $174 \AA$ band while the campfire is almost brightest side by side with the selected magnetic field lines. The three groups are (i) crossing bundles of field lines (three cases; sample in Figs. 4a and b), (ii) forking bundles of field lines (three cases; Figs. 4c and d), and (iii) highly twisted flux ropes (one case; Figs. 4e and $\mathrm{f}$ ).

The brightenings in group (i) are associated with two bundles of field lines. In the initial stages, these bundles are almost perpendicular to each other. The enhanced EUI $174 \AA$ emission predominantly originates from the interface between the two bundles (Figs. $4 \mathrm{a}$ and $\mathrm{b}$ ). In this case, the field-line bundle running along the $y$-direction is rising. Following the interaction between the two bundles, only the field lines of the bundle in the $x$-direction remain (see animation associated with Figs. $4 \mathrm{a}$ and $b$ ). This is indicative of reconnection, and in response to the heating the plasma gets hot enough to radiate in the $174 \AA$ band. The other two examples of this case are shown in Figs. 5a,b, Figs. A.1e,f, and the associated animations.

The brightenings of group (ii) are associated with bundles of forking field lines. Before their interaction, the two bundles originate from one common magnetic patch in the photosphere. The other ends of the bundles are rooted at two separate magnetic patches, leading to an overall appearance of a forking structure (see Figs. 4c and d). During the evolution, again illustrated in animation with the figure, the heating and subsequent brightening appear at the location where the two field-line bundles are separating, that is, form the fork. As in group (i), after the brightening, only one of the bundles of field lines remains. The other two examples of this group are shown in Figs. A.1a, b and Figs. A.1c, d.

The events in group (i) and (ii) are quite similar in terms of magnetic topology. Firstly, after each of these six events, we found that one bundle of magnetic field lines is almost completely eroded once the campfire disappears (see animations with the figures). This also underlines that reconnection is the energy source for the brightening. Secondly, the reconnection between the field lines involved is occurring at small angles of $90^{\circ}$ or less, that is, the reconnecting field lines are nearly perpendicular to each other or point roughly in the same direction. This suggests that the campfires are most likely 

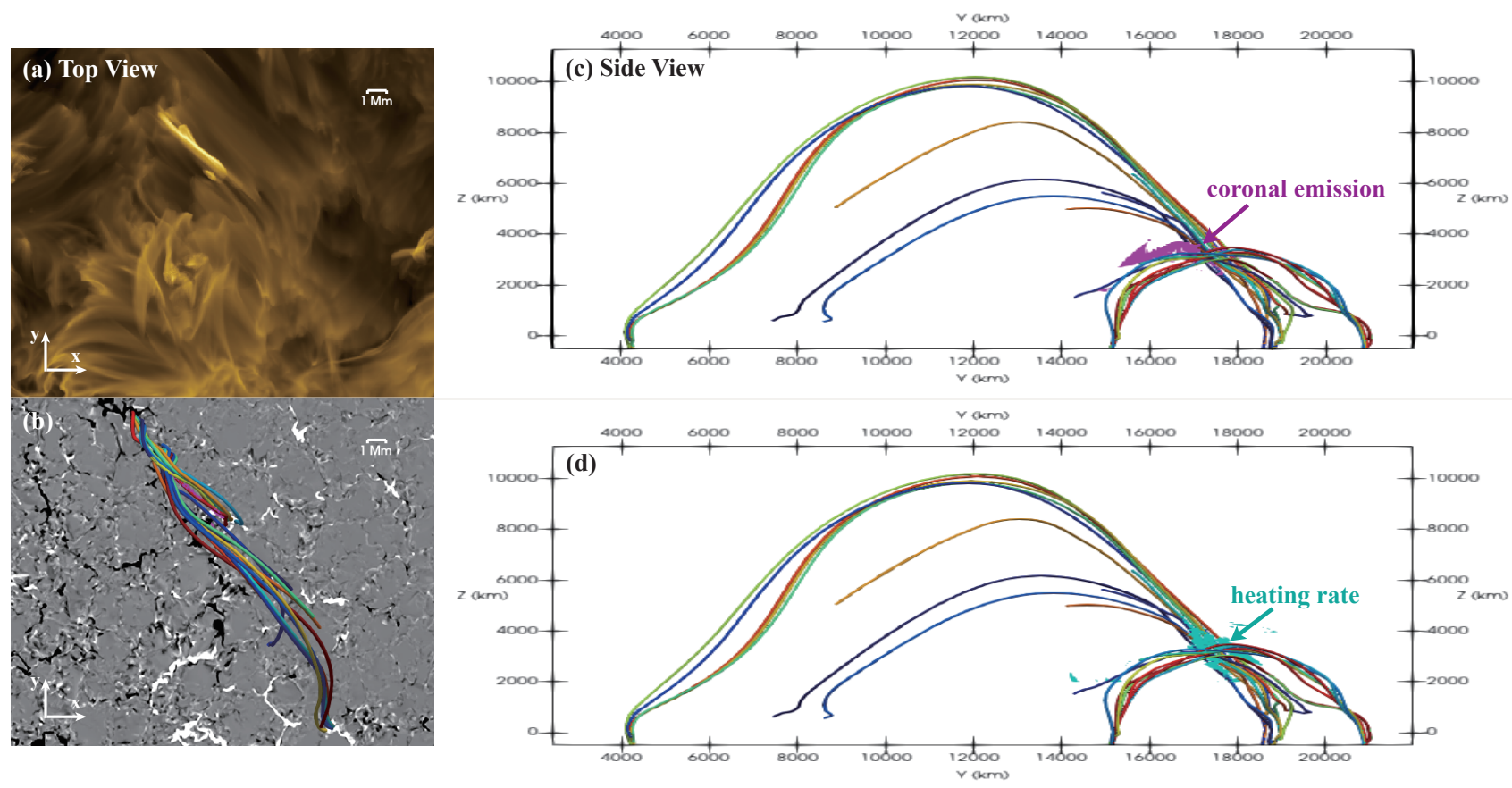

Fig. 5. Spatial appearance of campfires. Panels $a$ and $b$ : another example of a campfire in the same format as in Fig. 4 (at time $t=260 \mathrm{~s}$ ). Panels $c$ and $d$ : projection of the field lines in panel $\mathrm{b}$ onto the vertical $y-z$ plane. All the field lines are mostly in a vertical plane at a ca. $30^{\circ}$ angle to the y-direction. Therefore we scaled the side view in panels $c$ and $d$ so that the field lines appear at the approximately correct aspect ratio of the horizontal to vertical extent. In panel $c$ we overplot a mask of the EUI $174 \AA$ A emission in purple, and in panel $d$ the heating rate is overplotted (resistive plus viscous) in cyan. An animation of panel c is available online. See Sect. 3.3.

caused by component magnetic reconnection between different magnetic structures (e.g., Sonnerup 1974), a process otherwise mostly considered in open magnetic configurations such as coronal holes (e.g., Tenerani et al. 2016; Viall \& Borovsky 2020). This is unlike anti-parallel reconnection that was frequently seen in recent MHD simulations of explosive events and UV bursts (e.g., Innes et al. 2015; Ni et al. 2016, 2021; Priest et al. 2018; Syntelis et al. 2019; Peter et al. 2019; Danilovic 2017; Hansteen et al. 2017, 2019), where the angle between the directions of reconnecting magnetic field lines is generally about $180^{\circ}$.

For one example from groups (i) and (ii), we take a closer look at the relation of reconnection, heating, and brightening in the EUV. The campfire shown in Fig. 5a exhibits an elongated loop-like morphology. The magnetic field lines around the campfire show a very high degree of overlapping when viewed from the top (Fig. 5b). To better illustrate the magnetic field structures around the campfire, we show a projection onto the vertical $y z-$ plane. In this projection, the field lines from the two bundles show a crossing under small angles, as is common for the other group (i) and (ii) events. At the location where the two field-line bundles cross, we find an enhanced heating rate (resistive plus viscous), which underlines the presence of reconnection heating the plasma. This is illustrated in Fig. 5d and underlines the presence of component reconnection heating the plasma. The emission in the $174 \AA$ band will not be identical to the location of the heat input, of course. For example, if one of the two bundles of field lines has a lower density than the other, then we will mostly see the enhanced emission on the bundle where the (pre-event) density was higher. In the example in Fig. 5, this is the case for the lower-lying bundle. Because the field lines are, very roughly, semicircular (see Fig. 5) and the typical distance between opposite magnetic polarities in the quiet-Sun network is on the order of a few granules, we can typically expect these closed field lines to reach not much higher than $5 \mathrm{Mm}$ (e.g., Jendersie \& Peter
2006). Consequently, the campfires also mostly do not exceed this height, and the seven examples we analyze here are occurring at heights between $2 \mathrm{Mm}$ and $5 \mathrm{Mm}$. This height range for the campfires roughly matches the height range where the normal quiescent coronal emission in the $174 \AA$ band originates.

From this we conclude that for the events from groups (i) and (ii), component reconnection leads to heating of plasma in the vicinity of the reconnection region of the interacting field-line bundles. This causes the region near the top of one of the fieldline bundles to brighten. This supports one of the interpretations given in the observations by Berghmans et al. (2021) to explain why the length of the campfires is smaller than twice their height above the photosphere.

The single event in group (iii) is quite different in nature and obviously associated with an untwisting magnetic flux rope. At the location of the brightening both before and during the event, the field lines visualize a flux rope (Figs. 4e and f). The temporal evolution of the magnetic field structure shows the relaxation of this highly twisted flux rope (see animation associated with the figure). At the end of the brightening in the $174 \AA$ channel, the bundle of field lines is largely, but not completely, untwisted. It is possible that component magnetic reconnection between internal adjacent field lines within the flux rope (e.g., Xing et al. 2020) heats the plasma to $1 \mathrm{MK}$ or more and also results in a reduction of twist. Due to the small number of events investigated in this study, we cannot determine whether this mechanism makes a substantial fraction of campfires.

\section{Discussion}

Our results derived from the (synthesized) coronal emission seen in the transient brightenings show a good match to the campfires as reported in the observations by Berghmans et al. (2021). In 


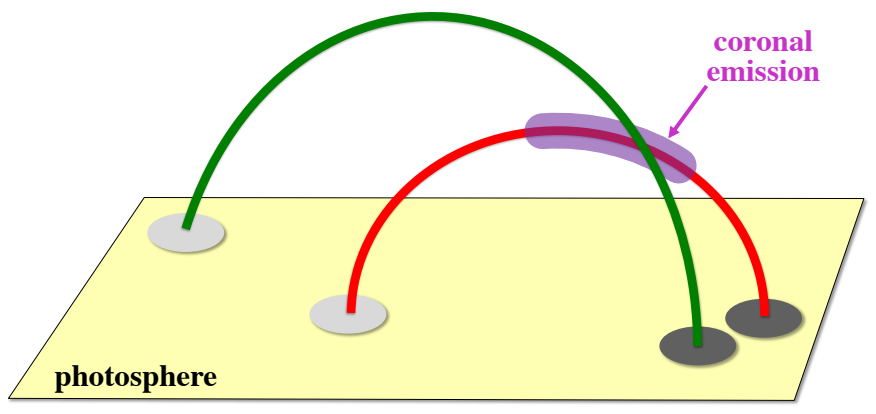

Fig. 6. Magnetic structure of a campfire. This illustrates the majority of the campfires in our model, i.e., those from groups (i) and (ii) defined in Sect. 3.2. The light and dark gray areas represent patches of opposite magnetic polarity in the photosphere where the two interacting bundles of field lines (red and green) are rooted. Following the reconnection, coronal emission is seen along a loop-like stretch (purple). Essentially, this follows the structure found in the model for the case shown in Fig. 5c. See Sect. 4.

Sect. 3.2, we detail how the seven items listed in the introduction are recovered by our model. This suggests that the mechanism(s) driving the transients in our model might resemble the physics governing the observed campfires, even though one should remember that the Sun might still choose a different path.

In our model, we find that in the majority of cases, the energization mechanism driving the transient brightening is the component reconnection in the coronal part of interacting bundles of field lines, viz. coronal loops. This is illustrated in Fig. 6. While heating is mostly restricted to the region where the field-line bundles interact, the brightening is visible through the heated plasma on the interacting loop bundles. In the cartoon in Fig. 6, we imply that the density, before interaction, was higher on one of the two field-line bundles, and consequently this dominates the emission pattern and forms a loop-like structure stretching away from the reconnection region. At the lower parts of the field-line bundles, the densities are high so that the released energy is not sufficient to heat the plasma there to $\sim 1 \mathrm{MK}$. As a result, the EUI $174 \AA$ emission is confined at the higher parts and it does not reach the footpoints of the field-line bundles. This magnetic and emission structure is quite similar to the transient brightening in the MHD model shown in Fig. 5c.

This type of magnetic interaction has been found before for active regions and flaring loops. The rearrangement of tangled loops has been found to be responsible for the heating of some compact hot active-region loops (Reale et al. 2019) and postflare loops (Parenti et al. 2010). Also examples of cool loops that stay well below $0.1 \mathrm{MK}$ have been found to be heated in response to interacting flux bundles ( $\mathrm{Li}$ et al. 2014). The processes in these loops would correspond to the campfires of group (i) and (ii) as defined in Sect. 3.2. The event in group (iii) might be more comparable to flux ropes as found in solar flares and coronal mass ejections characterized by highly twisted field lines (e.g., Shibata \& Magara 2011; Cheng et al. 2017). In this study, we find that there is one common aspect of these active-region features and the campfire events: The energy to heat these structures is deposited in the upper (coronal) part of the features, in some cases near the loop apex. In the case of the campfires, this energizing near the apex is consistent with the observation of them occurring at heights significantly above the photosphere. Furthermore, it is worth mentioning that component reconnection can generate mini-jets in prominence structures (Chen et al. 2017, 2020; Antolin et al. 2021).
In contrast to these campfires, for many if not most smallscale transients on the Sun, the energy is thought to be supplied through reconnection at the footpoints that accompanies flux emergence and cancellation. This applies to Ellerman bombs (e.g., Georgoulis et al. 2002; Schmieder et al. 2014), UV bursts (e.g., Peter et al. 2014; Tian et al. 2016, 2018; Li et al. 2018), and spicules (e.g., Yurchyshyn et al. 2013; Samanta et al. 2019). Small-scale coronal brightenings in the core of active regions are closely related to flux emergence (Tiwari et al. 2019). Even coronal loops, both regular and flaring, might be heated through reconnection at or near their loop footpoints (Chitta et al. 2017, 2018). A common feature of these events is flux emergence and/or cancellation. Unfortunately, for the campfire observations by Berghmans et al. (2021), no high-resolution magnetic field observations are available to establish if campfires are related to flux emergence or cancellation. In our model, we do not see signatures of flux emergence or cancellation in the photosphere around the campfires. Instead, the loops, or field-line bundles, are driven by horizontal motions in the photosphere and they then interact at coronal heights through component reconnection. Future magnetic field observations are required to show if this prediction of our model is correct, namely that campfires are not clearly related to flux emergence and cancellation. This also underlines the need for measurements of the coronal magnetic field (e.g., Li et al. 2015; Yang et al. 2020a,b; Landi et al. 2020).

Component magnetic reconnection naturally occurs in a scenario of braiding magnetic field structures (Parker 1983, 1988). Pioneering 3D MHD numerical experiments about magnetic braiding were already performed in the 1990s (e.g., Galsgaard \& Nordlund 1996). Since then, significant progress has been made in realistic simulations of coronal heating caused by footpoint braiding (e.g., Gudiksen \& Nordlund 2002; Bingert \& Peter 2011; Hansteen et al. 2015; Rempel 2017). Properties of small-scale heating events caused by the current dissipation in the corona have also been explored through 3D MHD simulations (e.g., Bingert \& Peter 2013; Guerreiro et al. 2015, 2017). However, direct possible observational evidence of braiding is sparse (Cirtain et al. 2013; Huang et al. 2018). According to our model, in many of these campfires, the angles between reconnecting field lines are small, with field lines generally pointing in a similar direction. Thus we might consider campfires as indirect evidence for this process.

In principle, an increase in the EUV emission in a particular wavelength band or spectral line originating at a given temperature can occur for several reasons: The plasma is heated from lower temperatures or is cooling from higher temperatures, or the density increases, for example, due to a flow into the structure or by compression through the magnetic field (e.g., Harrison 1997). The cause of EUV brightenings in the transition region has been debated, with a slight preference for density increase (e.g., Bewsher et al. 2003). For the coronal campfires, we see in our model that we can safely conclude that they are solely due to the heating of plasma on the reconnecting bundles of field lines.

Finally, we turn to the energetics of the campfire events in our model. We estimated the total dissipated energy (viscous plus resistive heating) during the campfire shown in Fig. 5. We first selected the snapshot when the campfire reached its peak intensity. Then, we integrated the heating rate at all pixels within the cyan patch in Fig. 5, where the heating rate is significantly enhanced. The total energy was found to be on the order of $10^{27}$ ergs. We also examined the other events and found similar results. Thus, the campfires might be categorized as microflares in terms of energy. The energy is nearly two orders of magnitude smaller than that of a typical UV 
burst (Peter et al. 2014), which likely occurs at a lower height of $\sim 1 \mathrm{Mm}$ above the photosphere (Tian et al. 2018; Chen et al. $2019 b)$. We identified seven events over the span of $16 \mathrm{~min}$ within an area of $50 \times 50 \mathrm{Mm}^{2}$, which corresponds to an average energy flux of roughly $3 \times 10^{5} \mathrm{erg} \mathrm{cm}^{-2} \mathrm{~s}^{-1}$. This supply is comparable to the canonical amount of energy required to heat the quiet-Sun corona (Withbroe \& Noyes 1977). Furthermore, additional campfire-like events would be identified if we were to lower the threshold for event detection. Considering this, we conjecture that campfires should play an important role in the energization of the quiet-Sun coronal plasma. Some observational studies suggest that bursty events in the quiet-Sun corona do not play a dominant role in the heating of the corona (e.g., Aschwanden et al. 2000; Chitta et al. 2021). Whether campfirelike events can maintain a hot corona on the real Sun is still unclear. Further observational studies, particularly with the superior spatial resolution of EUI/HRT on Solar Orbiter, and a more thorough investigation through numerical experiments are needed to settle this issue.

\section{Conclusions}

To understand the processes underlying the campfires recently observed with EUI (Berghmans et al. 2021), we employed a numerical 3D MHD model of the quiet Sun using the MURaM code. The model self-consistently creates the typical magnetic patterns of supergranulation with a million $\mathrm{K}$ hot corona above.

The transients we find in $174 \AA$ emission synthesized from our numerical model show properties that match those of observed campfires. They match in terms of lifetime, size, aspect ratio, temperature, and radiative losses; furthermore, they occur at the same height adjacent to, but not directly above, bright patches of the chromospheric network. With this, the transients in our model are in line with the seven items listed in the introduction that summarize the observational findings of campfires by Berghmans et al. (2021).

The MHD model contains the full information of the selfconsistent 3D evolution of the magnetic field. Unlike in the observations, we can thus investigate the details of the magnetic processes in and around the campfires. From this we conclude that the campfires are mostly caused by component reconnection between interacting bundles of magnetic field lines. The interaction of the bundles and thus the heating to $1 \mathrm{MK}$ or more and the subsequent brightening in the EUV typically occurs at heights of up to $5 \mathrm{Mm}$ and is slightly offset (horizontally) to the magnetic concentrations that define the bright patches of the chromospheric network. In one out of seven cases, we found that untwisting of a flux rope is responsible for the heating of looplike features, which in EUV very much resembles the other cases due to component reconnection. We do not find obvious signatures of flux emergence or cancellation in the photosphere in any cases. Still, in our model we find that these transient brightenings might supply a sufficient amount of energy to heat the quiet-Sun corona.

Future investigations of the statistical properties of these transient events will have to show if what we found in the model is indeed the same type of feature as the observed campfires. The good match between observed and modeled properties, for a limited number of cases, makes us rather confident that this will be the case.

Acknowledgements. This work is supported by NSFC grants 11825301, 11790304 and 12073004, the Strategic Priority Research Program of CAS (grant no. XDA17040507), and the Max Planck Partner Group program. Y.C. also acknowledges partial support from the China Scholarship Council and the International Max Planck Research School (IMPRS) for Solar System Science at the University of Göttingen during his stay at MPS. Solar Orbiter is a space mission of international collaboration between ESA and NASA with contributions from national agencies of ESA member states. EUI was conceived by a multinational consortium and proposed in 2008 under the scientific lead of Royal Observatory of Belgium (ROB) and the engineering lead of Centre Spatial de Liège (CSL). We thank Dr. L. P. Chitta and Feng Chen for helpful discussion. This project has received funding from the European Research Council (ERC) under the European Union's Horizon 2020 research and innovation programme (grant agreement No. 695075). We gratefully acknowledge the computational resources provided by the Cobra supercomputer system of the Max Planck Computing and Data Facility (MPCDF) in Garching, Germany. D. P. would like to thank A. Irwin (Free-EoS) and V. Witzke (MPS-ATLAS) for the fantastic open-source packages they provide.

\section{References}

Alpert, S. E., Tiwari, S. K., Moore, R. L., Winebarger, A. R., \& Savage, S. L. 2016, ApJ, 822, 35

Antolin, P., Pagano, P., Testa, P., Petralia, A., \& Reale, F. 2021, Nat. Astron., 5, 54

Aschwanden, M. J. 2019, New Millennium Solar Physics, 458

Aschwanden, M. J., \& Parnell, C. E. 2002, ApJ, 572, 1048

Aschwanden, M. J., Tarbell, T. D., Nightingale, R. W., et al. 2000, ApJ, 535, 1047

Barczynski, K., Peter, H., \& Savage, S. L. 2017, A\&A, 599, A137

Barczynski, K., Peter, H., Chitta, L. P., \& Solanki, S. K. 2018, A\&A, 619, A5

Bellot Rubio, L., \& Orozco Suárez, D. 2019, Liv. Rev. Sol. Phys., 16, 1

Berghmans, D., Clette, F., \& Moses, D. 1998, A\&A, 336, 1039

Berghmans, D., Auchère, F., Long, D. M., et al. 2021, A\&A, 656, L4 (SO Cruise Phase SI)

Bewsher, D., Parnell, C. E., Pike, C. D., \& Harrison, R. A. 2003, Sol. Phys., 215, 217

Bingert, S., \& Peter, H. 2011, A\&A, 530, A112

Bingert, S., \& Peter, H. 2013, A\&A, 550, A30

Carlsson, M., \& Leenaarts, J. 2012, A\&A, 539, A39

Chen, H., Zhang, J., Ma, S., Yan, X., \& Xue, J. 2017, ApJ, 841, L13

Chen, Y., Tian, H., Huang, Z., Peter, H., \& Samanta, T. 2019a, ApJ, 873, 79

Chen, Y., Tian, H., Peter, H., et al. 2019b, ApJ, 875, L30

Chen, H., Zhang, J., De Pontieu, B., et al. 2020, ApJ, 899, 19

Cheng, X., Guo, Y., \& Ding, M. 2017, Sci. China Earth Sci., 60, 1383

Chitta, L. P., Peter, H., Solanki, S. K., et al. 2017, ApJS, 229, 4

Chitta, L. P., Peter, H., \& Solanki, S. K. 2018, A\&A, 615, L9

Chitta, L. P., Peter, H., \& Young, P. R. 2021, A\&A, 647, A159

Cirtain, J. W., Golub, L., Winebarger, A. R., et al. 2013, Nature, 493, 501

Danilovic, S. 2017, A\&A, 601, A122

Delaboudinière, J. P., Artzner, G. E., Brunaud, J., et al. 1995, Sol. Phys., 162, 291

Del Zanna, G., Dere, K. P., Young, P. R., \& Landi, E. 2021, ApJ, 909, 38

De Pontieu, B., Title, A. M., Lemen, J. R., et al. 2014, Sol. Phys., 289, 2733

Dere, K. P., Bartoe, J. D. F., \& Brueckner, G. E. 1989, Sol. Phys., 123, 41

Dere, K. P., Landi, E., Mason, H. E., Monsignori Fossi, B. C., \& Young, P. R. 1997, A\&AS, 125, 149

Feldman, U. 1992, Phys. Scr., 46, 202

Galsgaard, K., \& Nordlund, A.. 1996, J. Geophys. Res., 101, 13445

Georgoulis, M. K., Rust, D. M., Bernasconi, P. N., \& Schmieder, B. 2002, ApJ, 575, 506

Gudiksen, B. V., \& Nordlund, Å. 2002, ApJ, 572, L113

Guerreiro, N., Haberreiter, M., Hansteen, V., \& Schmutz, W. 2015, ApJ, 813, 61

Guerreiro, N., Haberreiter, M., Hansteen, V., \& Schmutz, W. 2017, A\&A, 603, A103

Handy, B. N., Acton, L. W., Kankelborg, C. C., et al. 1999, Sol. Phys., 187, 229 Hansteen, V., Guerreiro, N., De Pontieu, B., \& Carlsson, M. 2015, ApJ, 811, 106 Hansteen, V. H., Archontis, V., Pereira, T. M. D., et al. 2017, ApJ, 839, 22 Hansteen, V., Ortiz, A., Archontis, V., et al. 2019, A\&A, 626, A33

Harra, L. K., Gallagher, P. T., \& Phillips, K. J. H. 2000, A\&A, 362, 371 Harrison, R. A. 1997, Sol. Phys., 175, 467

Howard, R. A., Moses, J. D., Vourlidas, A., et al. 2008, Space Sci. Rev., 136, 67 Huang, Z., Xia, L., Nelson, C. J., et al. 2018, ApJ, 854, 80

Innes, D. E., Inhester, B., Axford, W. I., \& Wilhelm, K. 1997, Nature, 386, 811 Innes, D. E., Guo, L. J., Huang, Y. M., \& Bhattacharjee, A. 2015, ApJ, 813, 86 Irwin, A. W. 2012, Astrophysics Source Code Library [record ascl:1211.002] Jendersie, S., \& Peter, H. 2006, A\&A, 460, 901

Kobayashi, K., Cirtain, J., Winebarger, A. R., et al. 2014, Sol. Phys., 289, 4393 Landi, E., Hutton, R., Brage, T., \& Li, W. 2020, ApJ, 904, 87

Lemen, J. R., Title, A. M., Akin, D. J., et al. 2012, Sol. Phys., 275, 17 
A\&A 656, L7 (2021)

Li, L. P., Peter, H., Chen, F., \& Zhang, J. 2014, A\&A, 570, A93

Li, W., Grumer, J., Yang, Y., et al. 2015, ApJ, 807, 69

Li, D., Li, L., \& Ning, Z. 2018, MNRAS, 479, 2382

Madjarska, M. S. 2019, Liv. Rev. Sol. Phys., 16, 2

Müller, D., St. Cyr, O. C., Zouganelis, I., et al. 2020, A\&A, 642, A1

Ni, L., Lin, J., Roussev, I. I., \& Schmieder, B. 2016, ApJ, 832, 195

Ni, L., Chen, Y., Peter, H., Tian, H., \& Lin, J. 2021, A\&A, 646, A88

Parenti, S., Reale, F., \& Reeves, K. K. 2010, A\&A, 517, A41

Parker, E. N. 1983, ApJ, 264, 642

Parker, E. N. 1988, ApJ, 330, 474

Peter, H., Bingert, S., Klimchuk, J. A., et al. 2013, A\&A, 556, A104

Peter, H., Tian, H., Curdt, W., et al. 2014, Science, 346, 1255726

Peter, H., Huang, Y. M., Chitta, L. P., \& Young, P. R. 2019, A\&A, 628, A8

Priest, E. R., Chitta, L. P., \& Syntelis, P. 2018, ApJ, 862, L24

Rachmeler, L. A., Winebarger, A. R., Savage, S. L., et al. 2019, Sol. Phys., 294, 174

Reale, F., Testa, P., Petralia, A., \& Graham, D. R. 2019, ApJ, 882, 7

Rempel, M. 2014, ApJ, 789, 132

Rempel, M. 2017, ApJ, 834, 10

Rochus, P., Auchère, F., Berghmans, D., et al. 2020, A\&A, 642, A8
Samanta, T., Tian, H., Yurchyshyn, V., et al. 2019, Science, 366, 890

Schmieder, B., Archontis, V., \& Pariat, E. 2014, Space Sci. Rev., 186, 227

Schüssler, M., \& Vögler, A. 2008, A\&A, 481, L5

Shibata, K., \& Magara, T. 2011, Liv. Rev. Sol. Phys., 8, 6

Sonnerup, B. U. Ö. 1974, J. Geophys. Res., 79, 1546

Syntelis, P., Priest, E. R., \& Chitta, L. P. 2019, ApJ, 872, 32

Tenerani, A., Velli, M., \& DeForest, C. 2016, ApJ, 825, L3

Tian, H., Kleint, L., Peter, H., et al. 2014, ApJ, 790, L29

Tian, H., Xu, Z., He, J., \& Madsen, C. 2016, ApJ, 824, 96

Tian, H., Zhu, X., Peter, H., et al. 2018, ApJ, 854, 174

Tiwari, S. K., Panesar, N. K., Moore, R. L., et al. 2019, ApJ, 887, 56

Viall, N. M., \& Borovsky, J. E. 2020, J. Geophys. Res.: Space Phys., 125, e26005

Vögler, A., Shelyag, S., Schüssler, M., et al. 2005, A\&A, 429, 335

Withbroe, G. L., \& Noyes, R. W. 1977, ARA\&A, 15, 363

Witzke, V., Shapiro, A. I., Cernetic, M., et al. 2021, A\&A, 653, A65

Xing, C., Cheng, X., Ding, M. D., \& The, 2020, The Innovation, 1, 100059

Yang, Z., Bethge, C., Tian, H., et al. 2020a, Science, 369, 694

Yang, Z., Tian, H., Tomczyk, S., et al. 2020b, Sci. China Technol. Sci., 63, 2357

Young, P. R., Tian, H., Peter, H., et al. 2018, Space Sci. Rev., 214, 120

Yurchyshyn, V., Abramenko, V., \& Goode, P. 2013, ApJ, 767, 17 


\section{Appendix A: Additional examples of campfires}

Our study investigated the seven strongest (viz. brightest) campfire events we found in our model. Based on their magnetic structure, we classified these into three groups (see Sect. 3.3 for the definition). In the main text, we show one example for each case in Fig. 4 and a further example in Fig. 5. Here we show the other three examples in the same format as in Fig. 4.
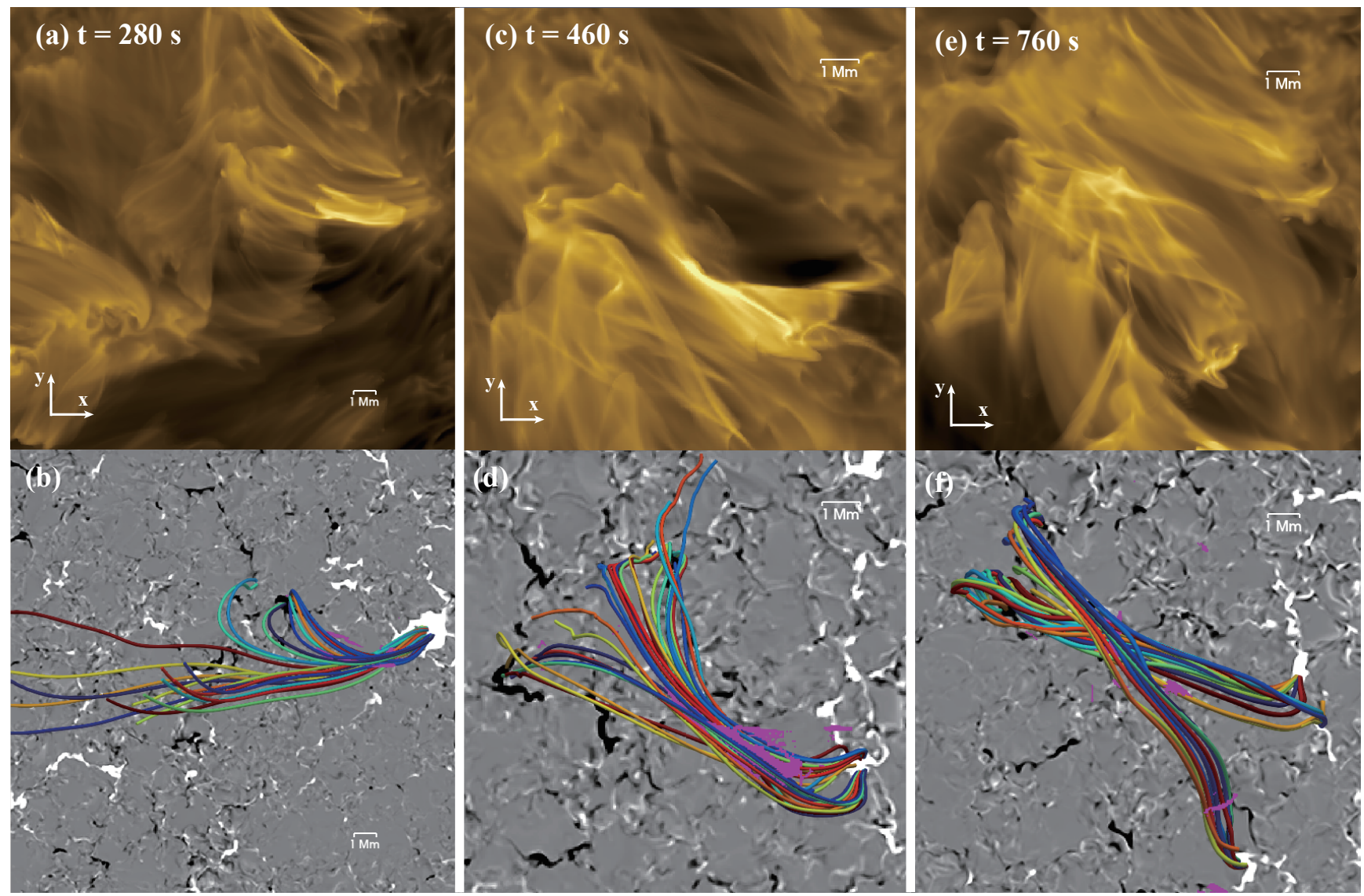

Fig. A.1. Relation of campfires to the magnetic field for three more examples. Same as Fig. 4, but for the cases not shown in the main paper in Figs. 4 and 5. Animations for each example are available online. 


\section{Appendix B: Lifetimes of the campfires}

For each of the seven campfires in our model, we examined the temporal variation of the EUI $174 \AA$ A emission. For this, we added up the intensity at full resolution over a small rectangle that contains the campfire. The resulting light curves for the seven cases are displayed in Fig. B.1. These light curves are not sensitive to the size of the rectangle because the campfires we selected dominate the $174 \AA$ emission locally. We also marked the times of the snapshots displayed in Figs. 4, 5, and A.1 that are close to peak intensity.

We used these light curves to estimate the lifetimes of the campfires. For this, we considered those snapshots (viz. times) as being part of the campfire for which the coronal brightening meets the criteria to identify campfires as described in Sect. 3.2. Some peaks in the curves were not identified as campfires because the spatial extent of the respective brightenings is smaller than our size criterion for campfire identification (cf. Sect. 3.2). Based on this, we indicated the duration of the campfires (in red). Typically, the lifetimes are below $3 \mathrm{~min}$.
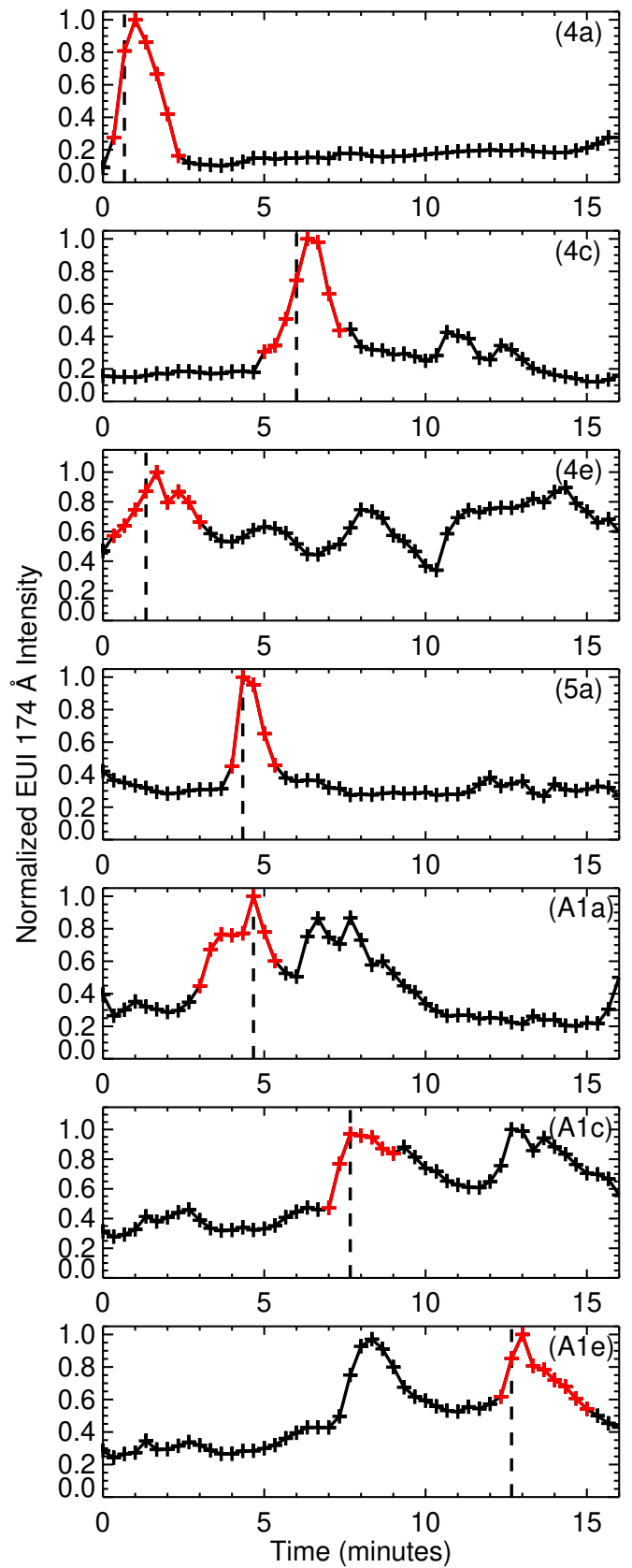

Fig. B.1. Light curves of modeled campfires. The panels show the temporal evolution in the EUI $174 \AA$ band as synthesized from our model. These campfires and their relation to the magnetic field are shown in detail in Figs. 4, 5, and A.1, as referred to by the labels in the top right of each panel. The red parts correspond to the periods when the campfires appear in the EUI $174 \AA$ images and the vertical dashed lines indicate the times of the snapshots in Figs. 4, 5, and A.1. See Appendix B. 